\title{
Simulation of the Thermal Aberration of Fused Silica Reflective Optics Under High-power Laser Irradiation
}

\section{Yuan Li}

Institute of applied electronics, CAEP

HongMing Yu

Institute of applied electronics, CAEP

XinQi Yin

Institute of applied electronics, CAEP

Juan Wu

Institute of applied electronics, CAEP

\section{Feng Wang}

Institute of applied electronics, CAEP

\section{Kai Zou}

Institute of applied electronics, CAEP

\section{Hong Yan}

Institute of applied electronics, CAEP

JianMin Li

Institute of applied electronics, CAEP

Jia Luo ( $\nabla$ tripor@163.com )

Institute of applied electronics, CAEP

\section{Research Article}

Keywords: thermal aberration, high-power laser, fused silica, thermal-structure simulation

Posted Date: September 15th, 2021

DOl: https://doi.org/10.21203/rs.3.rs-880767/v1

License: (c) (i) This work is licensed under a Creative Commons Attribution 4.0 International License.

Read Full License 
Simulation of the thermal aberration of fused silica reflective optics under high-power laser irradiation

Li Yuan, Yu HongMing, Yin XinQi, Wu Juan, Feng Wang, Zou Kai, Yan Hong, Li JianMin, Luo

Jia

Institute of applied electronics, CAEP

Correspondence should be addressed to L.J.M. (email: leejianmin@163.com) or L.J. (email:

$$
\text { tripor@163.com) }
$$

\section{Abstract}

The output beam quality of high-power laser systems is limited by laser-induced thermal aberration of fused silica reflective optics. A numerical model for the simulation of thermal aberration was proposed and verified by the experimental results. Simulations on the thermal aberration of fused silica optics under $3 \sim 10 \mathrm{~kW}$ laser irradiation with laser beam diameters of $5 \mathrm{~mm} \sim 45 \mathrm{~mm}$ were carried out with the verified model. The simulation results showed that the peak-valley $(\mathrm{PV})$ value of thermal aberration increases with increasing incident laser power under the same incident laser spot size and reduces with increasing incident laser spot size under the same incident laser power. There are the same PV values of thermal aberration under different incident power or power densities. An analytic formula of thermal aberration PV as a function of incident laser power and beam spot size was proposed. The analytic results are in good agreement with the simulations. With these conclusions, the thermal aberration of fused silica optics under high incident power and power density can be evaluated by that under low incident power and power density. It is helpful for the design of high-power laser systems to obtain reasonable output beam quality.

Key words: thermal aberration, high-power laser, fused silica, thermal-structure simulation

\section{Introduction}

The output beam quality of a high-power laser system is a crucial performance index [1-3]. It determines the laser power density at aim and the effect of the laser system. There is an optic transfer module that connects the high-power laser source and emission tower. The optic transfer module usually includes several fused silica reflective optics suffering high-power laser irradiation. Although these reflective optics were manufactured with high reflectivity (more than 99.99\%), a proportion of incident laser power was still absorbed by the high reflectivity films on the surface of fused silica optics [4]. Fused silica optics were heated by absorbed laser power, and aberrations were introduced by asymmetric heating [5-7]. The wavefront of the transfer laser was modulated by the thermal aberration of the transfer fused silica optics. Finally, it results in degradation of the output beam quality of high-power laser systems [8-10]. The evaluation of optical thermal aberrations is the basis of adaptive optics for aberration compensation [11-15] and is essential for the design of a high-power laser system with the desired output beam quality.

Developing simulation technology on optics-mechanics-thermodynamics multiphysics provides an excellent method to investigate the thermal aberrations of fused silica optics. Many thermal aberration simulation studies have been published [16-22]. In this work, a numerical model for thermal aberration simulation was built and verified by the experimental results. Then, efforts were made to investigate the influence of incident laser power, beam spot size and average power density on the thermal aberration of fused silica reflective optics. An experience formulation of thermal aberration PV as a function of incident laser power and beam spot size was also proposed. 


\section{Simulation}

1) Numerical model

The basic conception of this numerical model can be described as follows: a fused silica optic with high reflectivity film is irradiated by a high power laser beam. The optic is free to bound and surrounded by air at room temperature.

The temperature distribution of the fused silica optic is obtained by solving the unsteady heat exchange differential function:

$$
\rho \mathrm{C}(\mathrm{T}) \cdot \partial \mathrm{T} / \partial \mathrm{t}+\nabla \cdot(-\mathrm{K}(\mathrm{T}) \nabla \mathrm{T})=\mathrm{Q}
$$

$\mathrm{T}$ is a function of the temperature distribution, $\rho$ is the density of fused silica, $\mathrm{C}(\mathrm{T})$ is the heat capacity at constant pressure, $\mathrm{K}(\mathrm{T})$ is the thermal conductivity, $\mathrm{t}$ is the irradiation time, and $\mathrm{Q}$ is the thermal source.

There are two kinds of laser thermal sources in this numerical model: surface thermal sources and volume thermal sources. A surface thermal source is generated by absorption of the film to the incident laser, with the following format:

$$
\mathrm{Q}_{\text {sur }}=\alpha \mathrm{I}
$$

$\alpha$ is the absorption coefficient of the film to the incident laser, and I is the power density function of the incident laser. The laser thermal source has the format of a Gauss function.

The volume thermal source is due to the absorption of fused silica volume material and can be expressed as:

$$
\mathrm{Q}=\beta(1-\mathrm{R}) \operatorname{Iexp}(-\beta \mathrm{z})
$$

$\beta$ is the absorption coefficient of the volume material to the incident laser, $\mathrm{R}$ is the reflectivity of fused silica reflecting optics, and $\mathrm{z}$ is the propagating distance in the volume material.

Then, the structural stress and deformation are generated by the results of the temperature distribution and by the coefficient of thermal expansion.

2) Model verify

Experimental and simulation tests were carried out to verify the simulation model. The setup of experiment is shown in figure 1. A fused silica sample with a $99.99 \%$ reflectivity film with a size of $\Phi 50 \mathrm{~mm} \times 5 \mathrm{~mm}$ was irradiated by a $3 \mathrm{~kW}$ laser with a beam spot diameter of $8 \mathrm{~mm}$. Laser power was measured by a power meter. The surface temperature and morphology were measured by a thermal camera and Shack-Hartmann sensor, respectively.

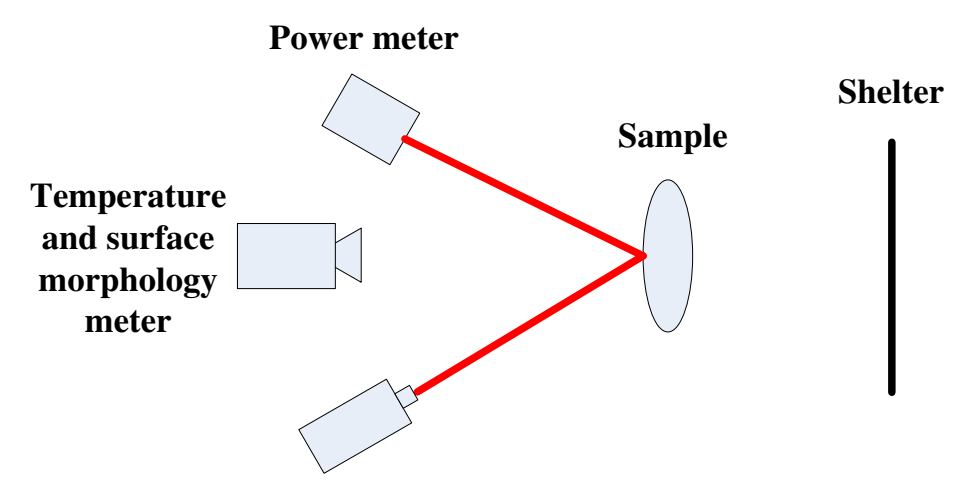

Figure 1 the sketch of thermal aberration experiment

The results showed that the surface temperature rose from $20^{\circ} \mathrm{C}$ to $25^{\circ} \mathrm{C}$, and the PV of thermal aberration was $5 \mathrm{~nm}$.

An FEM model was built in the EasyLaser simulation software (EasyLaser is a trademark of 
Institute of Applied Physics and Computational mathematics), which provides an integrated optomechanical analysis method and a GUI interface. The FEM model of the system includes 39002 domain elements. The conditions of initialization and boundary were consistent with the experiment. The parameters of the material and conditions are shown in Table 1.

Table 1 Simulation parameters of the material and conditions

\begin{tabular}{lll}
\hline Property & Value & Unit \\
\hline Young' modulus & $72 \mathrm{e} 9$ & $\mathrm{~Pa}$ \\
Poisson's ratio & 0.17 & 1 \\
Density & 2200 & $\mathrm{~kg} / \mathrm{m}^{\wedge} 3$ \\
Thermal conductivity & 1.4 & $\mathrm{~W} /(\mathrm{m} \cdot \mathrm{K})$ \\
Heat capacity at constant pressure & 840 & $\mathrm{~J} /(\mathrm{kg} \cdot \mathrm{K})$ \\
Coefficient of thermal expansion & $0.56 \mathrm{e}-6$ & $1 / \mathrm{K}$ \\
Initial temperature & 20 & ${ }^{\circ} \mathrm{C}$ \\
Heat transfer coefficient & 5 & $\mathrm{~W} /\left(\mathrm{m}^{\wedge} 2 \cdot \mathrm{K}\right)$ \\
Constrains & Free & 1 \\
\hline
\end{tabular}

Figure 2 shows the incident laser power distribution, the mesh of the FEM model and a set of results. The simulation results are in agreement with the experiment: after 30 seconds irradiation, the surface temperature of the sample is raised from $20^{\circ} \mathrm{C}$ to $25.7^{\circ} \mathrm{C}$, and the $\mathrm{PV}$ of thermal aberration in spot size is $4.5 \mathrm{~nm}$.

(a)
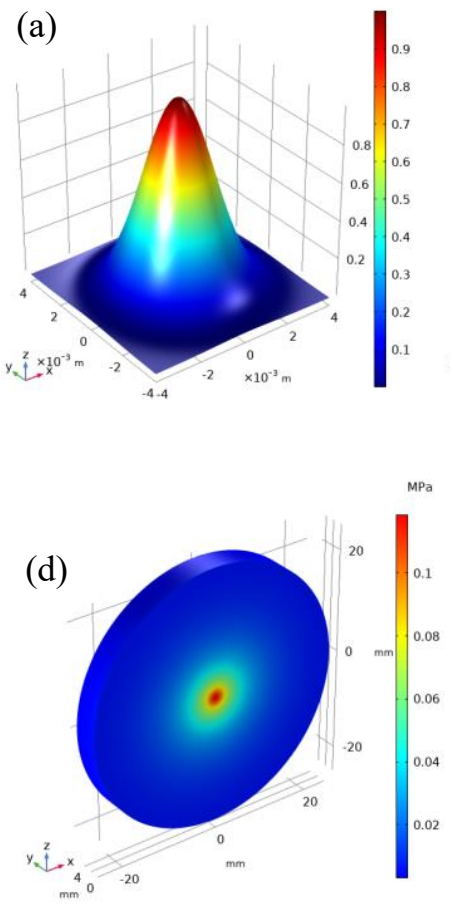

(b)

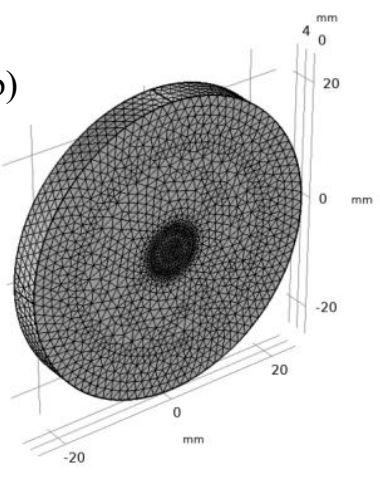

(e)

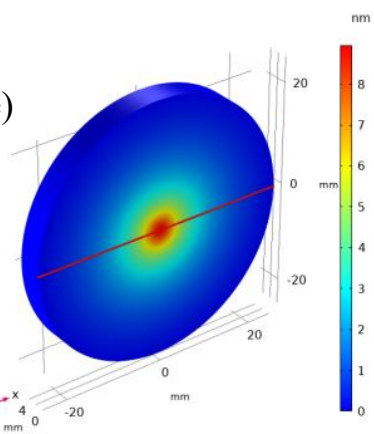

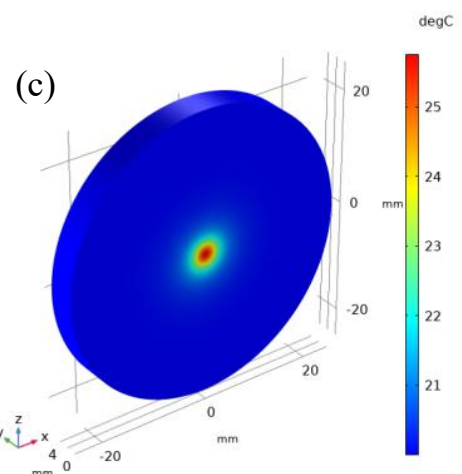

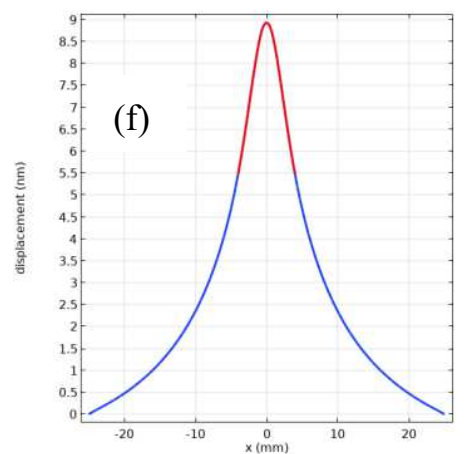

Figure 2 (a) Incident laser power distribution, (b) Mesh of the FEM model, (c) Temperature distribution, (d) and (e) Stress and displacement distribution, (f) profile of surface displacement. The agreement of the experiment and simulation shows that the numerical model of fused silica thermal aberration is reliable. Based on this verified numerical model, a simulation of the thermal aberration of fused silica reflective optics under high-power laser irradiation was carried out to 
explore the influence of incident power, beam spot size and power density on thermal aberration.

\section{Results}

1) Influence of incident power

The simulation results of the temperature and stress distributions at incident laser powers of $3 \mathrm{~kW}$ to $10 \mathrm{~kW}$ and beam sizes of $45 \mathrm{~mm}$ are shown in Figure 3 and Figure 4, respectively. The distribution forms of temperature and stress are the same at different incident powers, but only the values change. Figure 5 shows the profiles of the temperature and displacement distributions. The morphology of the temperature distribution is close to that of the incident laser power. The maximum value of the temperature increase is located at the center of the sample due to the Gaussian distribution of the incident laser power and low thermal conductivity of the fused silica material, while the temperature at the edge hardly rises. Accordingly, the morphology of the displacement distribution is high at the center and low close to the edge. The PV value of the surface thermal aberration increases from 4 $\mathrm{nm}$ to $13 \mathrm{~nm}$ as the incident laser power rises from $3 \mathrm{~kW}$ to $10 \mathrm{~kW}$. As seen in Figure 6, the PV value of the surface thermal aberration is directly proportional to the incident laser power.

The output beam quality of a high-power optic system is strongly dependent on the thermal aberration of the optics. Therefore, the results indicate that there is a limit of incident laser power given the size of the fused silica sample to obtain a designed output beam quality of a high-power optic system.

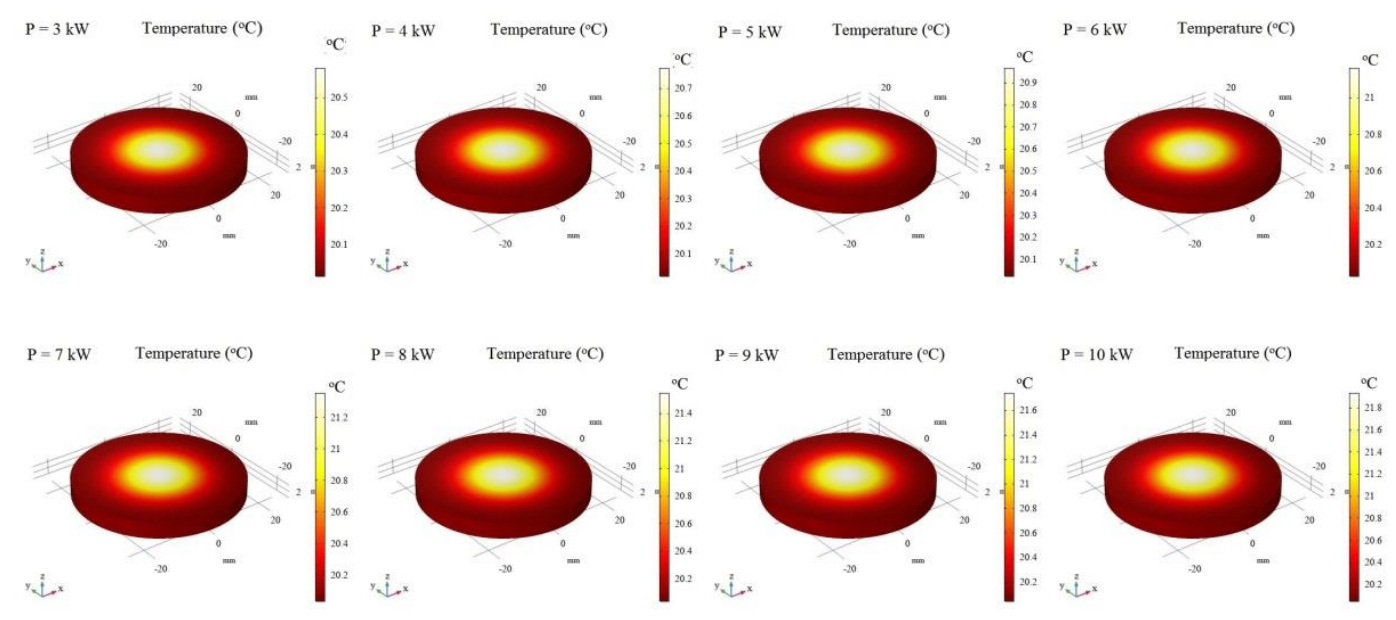

Figure 3 Temperature distributions at incident laser powers of $3 \mathrm{~kW}$ to $10 \mathrm{~kW}$.
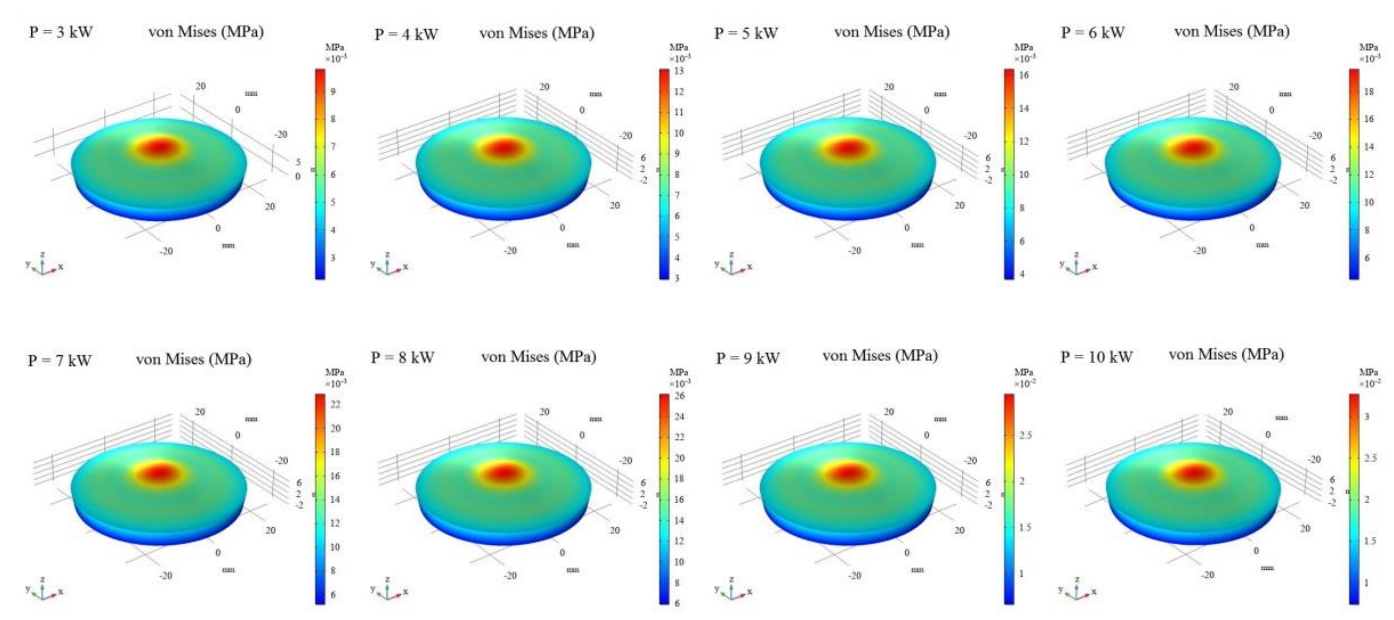

Figure 4 Stress distributions at incident laser powers of $3 \mathrm{~kW}$ to $10 \mathrm{~kW}$. 

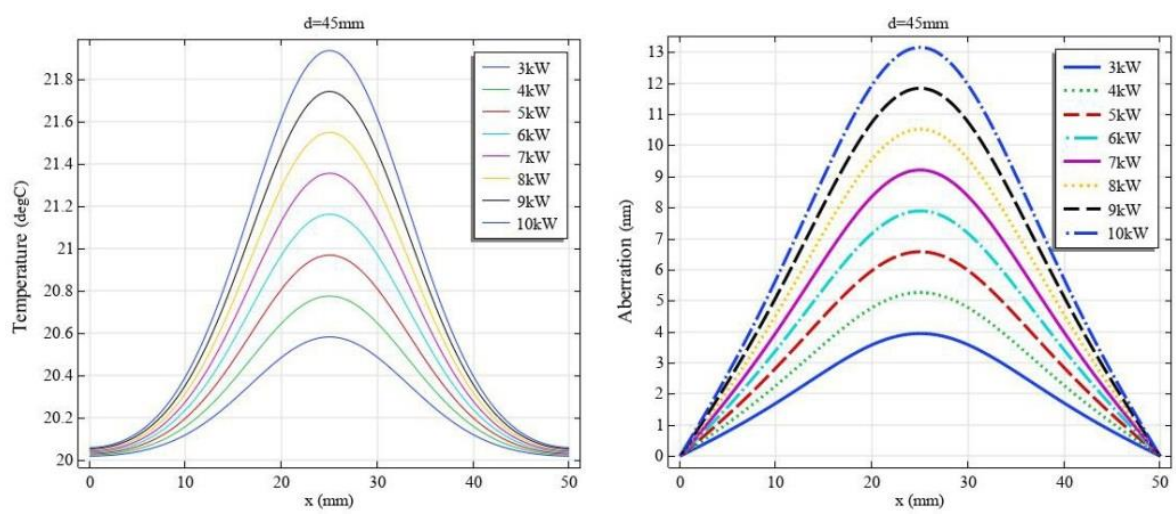

Figure 5 Profiles of temperature and displacement distributions

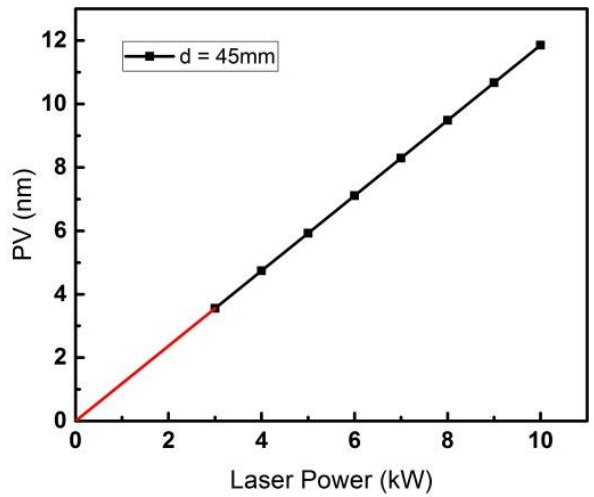

Figure 6 Thermal aberration as a function of incident laser power

2) Influence of incident beam spot size

Figure 7 and figure 8 show the simulation results of temperature and stress distributions with incident beam spot sizes from $5 \mathrm{~mm}$ to $40 \mathrm{~mm}$ at an incident laser power of $10 \mathrm{~kW}$, respectively. Figure 9 shows the surface profiles of the temperature and stress distributions. As seen in these figures, the distribution of temperature and stress are strongly dependent on the incident beam spot size. The scales of temperature rise and displacement increase with increasing incident beam spot size, while the maximum value of temperature and the PV value of surface displacement decrease as the incident beam spot size increases. This may be because the local power density is higher at the smaller beam spot size than at the larger beam spot size.

For a given incident laser power, increasing the incident beam spot size is helpful to decrease the thermal aberration of fused silica optics. In this simulation, the PV value of the thermal aberration decreases from $45 \mathrm{~nm}$ to $13 \mathrm{~nm}$ as the incident beam spot size increases from $5 \mathrm{~mm}$ to $40 \mathrm{~mm}$. The $\mathrm{PV}$ value of the surface thermal aberration is inversely proportional to the square of the incident laser beam size, as shown in Figure 10.
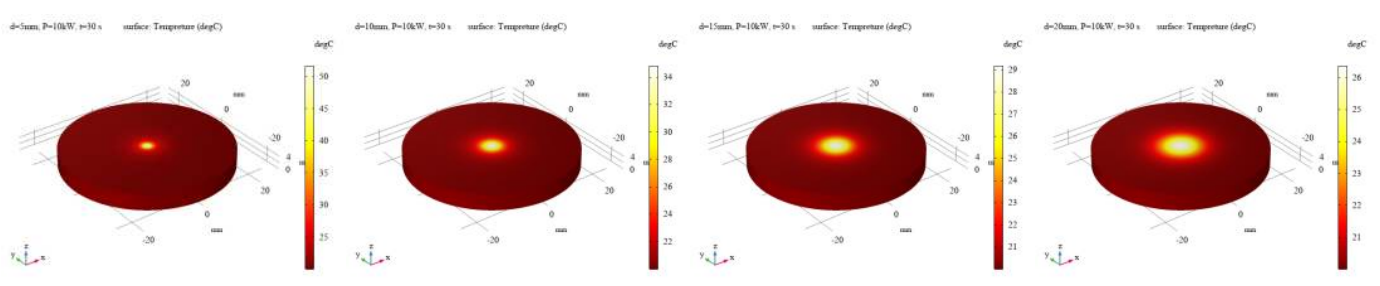


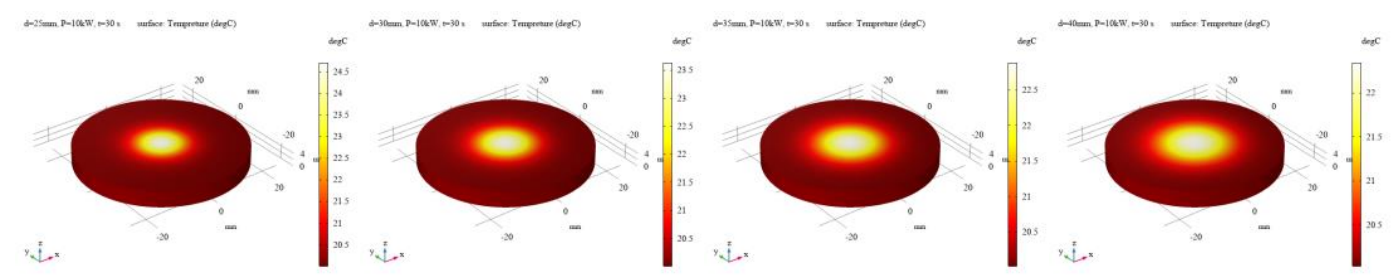

Figure 7 Temperature distributions with incident beam spot sizes from $3 \mathrm{~kW}$ to $10 \mathrm{~kW}$.
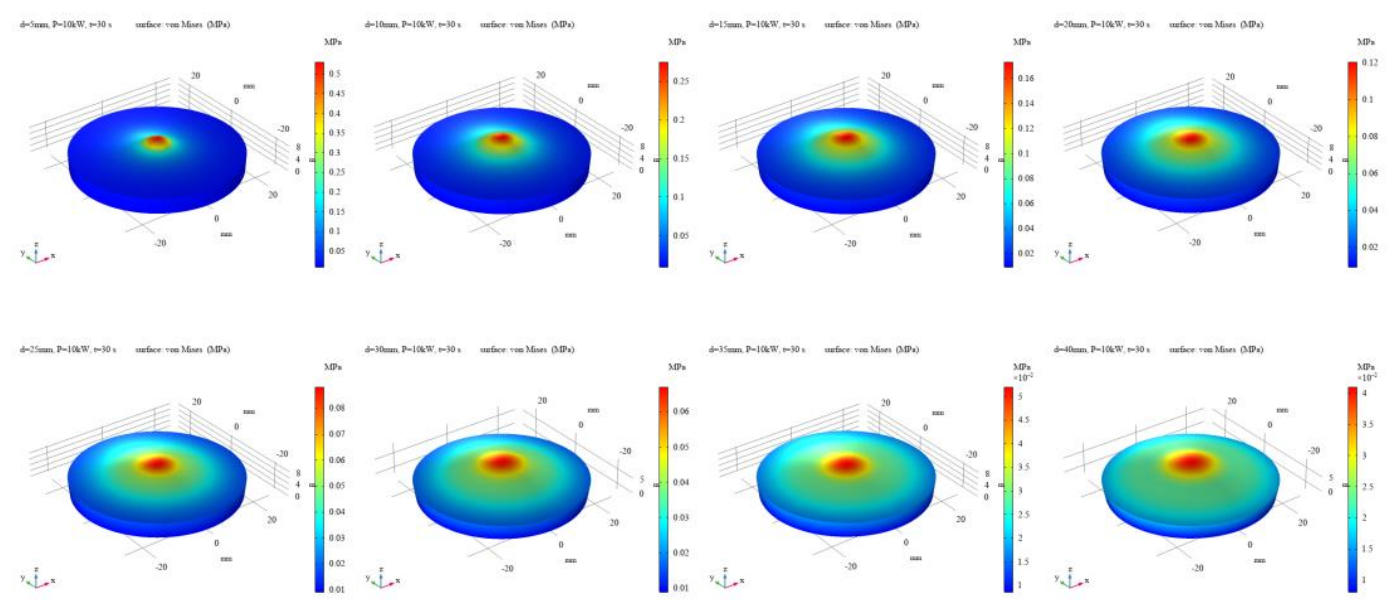

Figure 8 Stress distributions with incident beam spot sizes from $3 \mathrm{~kW}$ to $10 \mathrm{~kW}$.
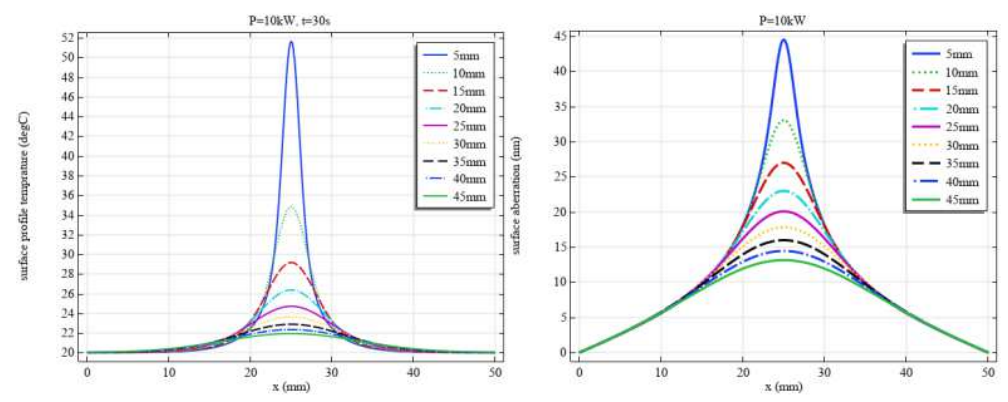

Figure 9 Profiles of temperature and displacement distributions

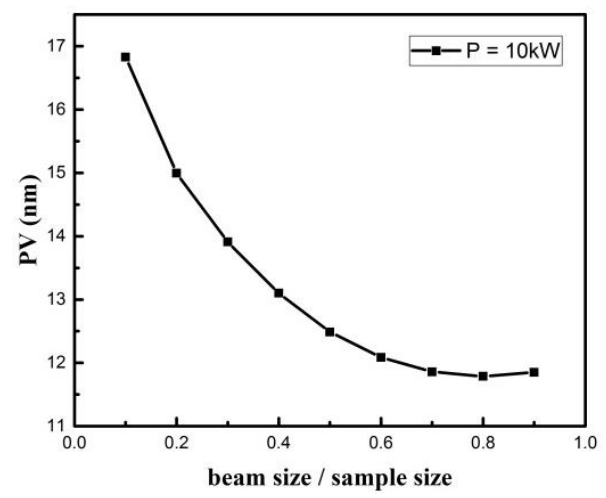

Figure 10 Thermal aberration as a function of incident beam spot size 3) Influence of incident average power density

Simulations with beam spot diameters from $5 \mathrm{~mm}$ to $45 \mathrm{~mm}$ at irradiation laser power from $3 \mathrm{~kW}$ to $10 \mathrm{~kW}$ were carried out to explore the influence of the incident average power density on the thermal aberration. The results are shown in figure 11. This is consistent with the discussions above: 
the PV value of the surface thermal aberration is directly proportional to the incident laser power for a given incident beam spot size and inversely proportional to the square of the incident laser beam size at a given incident laser power. However, the PV value of the surface thermal aberration is not directly proportional to the incident average power density. The PV value of the surface thermal aberration may be the same at different incident average power densities. According to these discussions, the influence of the incident average power density on the thermal aberration may be supposed with the following form:

$$
\mathrm{PV}=\mathrm{P} \cdot\left(\mathrm{A} \cdot r^{-2}+\mathrm{B} \cdot r^{-1}+C\right)
$$

where $\mathrm{PV}$ is the $\mathrm{PV}$ value of the surface thermal aberration, $\mathrm{P}$ is the incident laser power, $\mathrm{r}$ is the incident beam spot diameter, and $\mathrm{A}, \mathrm{B}$ and $\mathrm{C}$ are constants dependent on the fused silica material. In this simulation, the constants $\mathrm{A}, \mathrm{B}$ and $\mathrm{C}$ are calculated as $-0.0067,0.1320$ and 1.0326, respectively. Figure 12 compares the analytic and simulative results. It is in good agreement with each other. The proposed equation is useful to evaluate the magnitude of thermal aberration at high laser power irradiation according to the experimental results at low laser power. Moreover, it could be used to find the equivalent thermal aberration at different incident laser powers with various incident beam spot sizes.

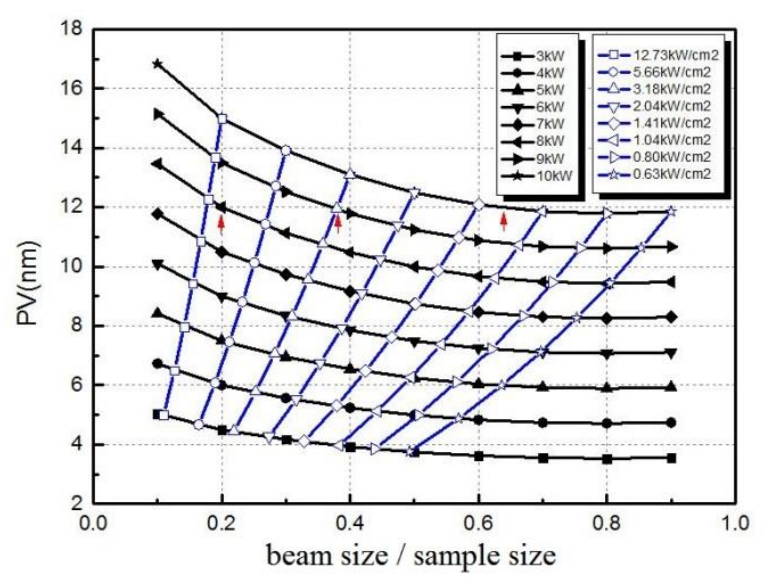

Figure 11 Influence of the incident average power density on the thermal aberration
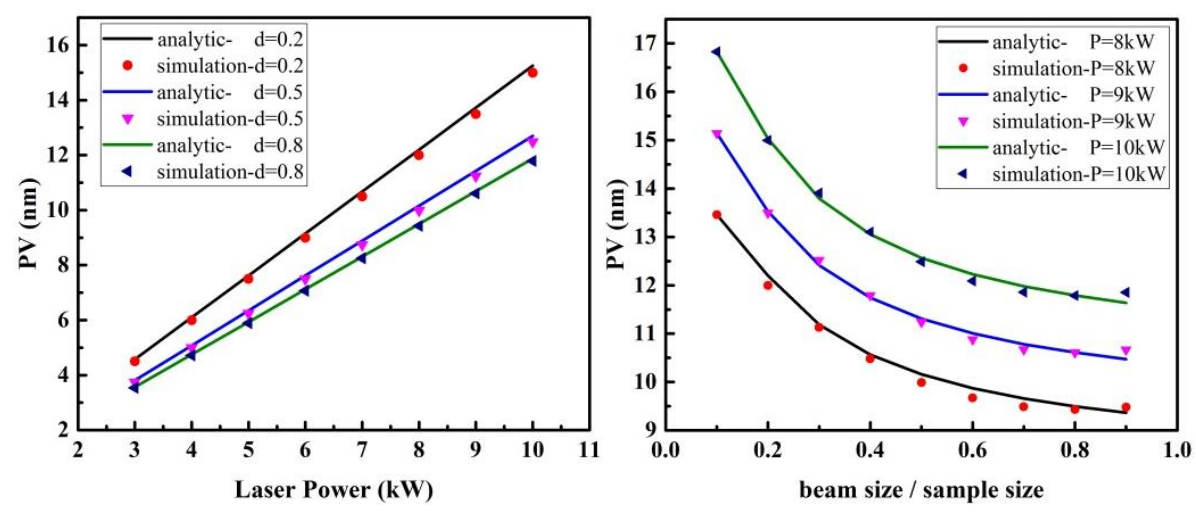

Figure 12 compares of analytic and simulation

\section{Conclusions}

In summary, a numerical model for the thermal aberration of fused silica reflective optics was built, and the simulation was in good agreement with the experimental results. The influence of incident power, beam spot size and average power density on thermal aberration was simulated based on the verified numerical model. The PV value of surface thermal aberration increases from $4 \mathrm{~nm}$ to 13 
$\mathrm{nm}$ as incident laser power rises from $3 \mathrm{~kW}$ to $10 \mathrm{~kW}$ with $45 \mathrm{~mm}$ incident beam spot diameter, but decreases from $45 \mathrm{~nm}$ to $13 \mathrm{~nm}$, as the incident beam spot size increases from $5 \mathrm{~mm}$ to $40 \mathrm{~mm}$ under $10 \mathrm{~kW}$ laser power irradiation. The results showed that the PV value of the thermal aberration is directly proportional to the incident laser power but inversely proportional to the square of the incident laser beam size, while the PV value of the thermal aberration is not proportional to the incident laser power density. Finally, the analytic function of the thermal aberration PV value dependent on the incident laser power and beam size was proposed and compared with the simulation. The analytic solution is consistent with the simulation. These conclusions are beneficial to evaluate the thermal aberration of fused silica reflective optics under high-power laser irradiation, in the design and usage of high--power laser systems to obtain reasonable output beam quality.

\section{Reference}

[1] Su Y., Wan M., High Power Laser System (in Chinese). (National defense industry press, 2003). [2] Du X., Factors for Evaluating Beam Quality of a Real High Power Laser on the Target Surface in Far Field. Chinese Journal of Lasers, 24(4),327-332 (1997).

[3] Wan M., Su Y., Zhang W., Influence of Initial Intensity Distribution on Laser Energy Concentration in the Far Field. Chinese Journal of Lasers, A29, 343-345 (2002).

[4] Qi W., Zhang Y., Thermal Absorption of Multilayer Dielectric Coatings. Opto-Electronic Engineering, 28(6), 33-36 (2001).

[5] Vincent L., Spencer W. J., Matthias S., Timo E., Sören J., Manuel K., Philipp M., Christian W., Paul W., Andreas R. M., Wavefront degradation of a 200 TW laser from heat-induced deformation of in-vacuum compressor gratings. Opt. Express, 26, 13061-13071 (2018)

[6] Steven T. Y. Manyalibo J. M., Selim E., Vaughn G. D., Scott E. B., Thermal transport in CO2 laser irradiated fused silica: In situ measurements and analysis. Journal of Applied Physics, 106,103106 (2009).

[7] Dai W., Xiang X., Jiang Y., Wang H.J., Li X.B., Yuan X.D., Zheng W.G., Lv H.B., Zu X.T., Surface evolution and laser damage resistance of $\mathrm{CO} 2$ laser irradiated area of fused silica. Optics \& Lasers in Engineering, 49(2), 273-280 (2011)

[8] Wan M., Su Y., Zhang W., Ye Y., Influence of the Surface Error of Optical Elements on Beam Quality. Acta Optica Sinica, 22(4), 495-500 (2002).

[9] Yuntao Q., Lei H., Mali G., Liu Q., Ping Y., Haitao Z., Method to evaluate beam quality of Gaussian beams with aberrations. Applied Optics, 51(27), 6539-6543(2012).

[10] Si C., Chi F., Yuan D., Jin G., Experimental study on spherical aberration and beam quality factor of LD end-pumped laser. Optics Communications, 467, 125627 (2020).

[11] Oughstun K. E., Intracavity adaptive optic compensation of phase aberrations. I: analysis. $J$. Opt. Soc. Am., 71, 862-872 (1981).

[12] Oughstun K. E., Spinhirne J. M., Anafi D., Intracavity adaptive optics. 4: comparison of theory and experiment. Appl. Opt., 23, 1529-1541 (1984).

[13] Cherezova T. Y., Kaptsov L. N., Kudryashov A. V., Cw industrial rod YAG:Nd3+ laser with an intracavity active bimorph mirror. Appl. Opt., 35, 2554-2561 (1996).

[14] Greiner U., Klingenberg H., Thermal lens correction of a diodepumped Nd:YAG laser of high TEM00 power by an adjustablecurvature mirror. Opt. Lett., 19, 1207-1209 (1994).

[15] Goebel T. A., Voigtländer C., Krämer R. G., Richter D., Heck M., Siems M. P., Matzdorf C., Reinlein C., Appelfelder M., Schreiber T., Thomas J. U., Tünnermann A., Nolte S., Flexible 
femtosecond inscription of fiber Bragg gratings by an optimized deformable mirror. Opt. Lett., $\mathbf{4 2}$, 4215-4218 (2017).

[16] Tao X., Ji X., Lv B., Numerical simulation of the thermal effect in a beam control system. Laser Technology, 27(6), 514-516 (2003).

[17]An J., Li Y., Du X., Influence of laser windows thermal lensing effect on beam quality. High Power Laser and Particle Beams, 16(4), 429-433 (2004).

[18] Hu X., Peng J., Zhang B., Thermal distortion of deformable mirror and its influence on beam quality. Chinese Journal of Lasers, 42(1), 0102003 (2015).

[19] Dzitoev A. M., Lapovok E. V., Khankov S. I., Thermal aberration of an off-axis mirror caused by temperature dropoff over its thickness. Journal of Optical Technology, 84(8), 542-547 (2017).

[20] Meng L., Huang Z., Han Z., Shen H., Zhu R., Simulation and experiment studies of aberration effects on the measurement of laser beam quality factor (M2). Optics and Lasers in Engineering, 100, 226-233 (2018).

[21] Zhang J., Chen F., Ma J., Pan S., Wei C., Wang M., Liu D., Thermal deformation of fused silica substrates and its influence on beam quality. Laser Technology, 43(3), 374-379 (2019).

[22] Li R., Yao P., Meng H., Wang J., Zhang K., Huang C., Zou B., Liu H., Coupled Thermalmechanical Analysis of CO2 Laser Irradiation on Fused silica. Advanced Materials Research, 1136, 531-536 (2016).

Author Contributions

Y.X.Q., W.F., Z.K. and Y.H. developed and directed the project. Y.L., Y.H.M. and L.J.M. conducted the experiment(s). Y.L., W.J. and L.J. analyzed the results help with writing the paper. All authors reviewed the manuscript.

Additional Information

Competing Interests: The authors declare that they have no competing interests. 